\section{P209 A PILOT QUALITY IMPROVEMENT (QI) INITIATIVE TO IMPROVE THE DISCHARGE PLANNING PROCESS FOR PATIENTS WITH COMPLEX CARE NEEDS AT TEMPLE STREET CHILDREN'S HOSPITAL (TSCUH), DUBLIN}

Shashi Vaish*, Gráinne Power, Claire Fagan, Elaine Fitzgerald, Sharon Ryan. TSCUH, Dublin, Ireland

10.1136/archdischild-2019-epa.564

Background Improvements in medical care have resulted in increasing numbers of children with complex care needs. These patients have substantial care needs resulting in functional limitations that may require tailored technological assistance (Cohen et al. 2011). These increased medical needs can result in prolonged hospital stay with a significant impact on the lives of the patient and their family. Discharge planning is an important aspect of patient care and can be challenging to organize for these patients. Currently there is a lot of disparity in the co-ordination of discharge or transfer process for these patients throughout our hospital. The has had a negative impact, some of which include, a delay in recognizing these patients and their needs, poor communication between health care professions, duplication of work and poor patient experience.

Aims A pilot QI initiative to improve the discharge planning process for long stay children with complex care needs by decreasing the time to initiate the discharge planning from more than 100 days to less than 60 days of in-patient stay on a ward at Temple Street Children's University Hospital, Dublin.

Methods Baseline data was collected and analysed to establish the median number of days before discharge planning was initiated for five children with complex care needs who were long-stay in-patients (i.e. for more than 30 days) on a particular ward at TSCUH. A patient-centred approach to improving inpatient discharge planning process for long-stay patients with complex care needs was created. A number of plan, do, study and act (PDSA) cycles were conducted in developing a baseline data collection method and a criteria based referral pathway to allow for initiation of a standardised discharge process for these patients. Early recognition of these patients assessed the impact on initiating the discharge planning process for patients admitted to one of the wards at TSCUH.

Results/Findings Data collection and collation is on-going and should be complete by March 2019 .

\section{P210 CHILD RIGHTS KNOWLEDGE AND UNDERSTANDING AMONG RUSSIAN CHILDREN AND ADOLESCENTS}

\footnotetext{
${ }^{1,2}$ Anastasia Rykunova* ${ }^{3}$ Maria Slipka, ${ }^{3}$ Maxim Strekalovsky, ${ }^{4,3}$ Sergey Slipka. ${ }^{1}$ Sechenovsky University, Moscow, Russian Federation; ${ }^{2}$ Union of Pediatricians of Russia, Moscow, Russian Federation; ${ }^{3}$ Union of Pediatricians of Russia, Moscow, Russian Federation; ${ }^{4}$ Central Clinical Hospital RAS, Moscow, Russian Federation

\subsection{6/archdischild-2019-epa.565}

Background Child Rights Convention (CRC) was ratified already 40 years ago and UN members states reported that changed and harmonised their legislation in accordance to CRC. However it is clear for pediatricians from the routine practice that there is a lack of informtion among child population about CRC and child rights implementation related to their health and development.
}

The aim of our study was to define how well children and adolescents (10-18 yo) of modern Russia are informed about their rights. We used our book 'CRC in the fairtales of the world' (ISSN 978-5-906332-81-3) to discuss with children and adolescents some articles of CRC related to general principles (articles 2-3-6-12), disability, basic health and wellfare (articles 23-24-26-27) and partially - family environment and alternative care (articles 18-20-21-25). The process included 3 days discussions with children and adolescents (with 4 discussions of each article during 30-45 Min - totally 120-180 min/day and 3 breaks for tea and snacks). Participants were randomly recruited in 4 schools of Moscow and Moscow's region. All children and their caregivers signed before the start of the study.

Results there was no difference among groups (15-17-19 persons) in average age $(12,783 \pm 0,043)$ or gender and sex distribution. All children under 13 were non-informed about their rights and the implementaton necesssity of CRS into real practice. Other respondents (from 14 to 18 ) was very critical to State, Gouvernement and even schools/medical doctors and mass media. No one from the groups was discussing the role of parents/caregivers/families in spreading this information (passive type of behavior).

Conclusion Further education of children and adolescents in the field of their rights implementation is needed.

\section{P211 CLINICAL BURDEN OF BOCAVIRUS INFECTION IN HOSPITALIZED CHILDREN}

Irina Chistol* , Zachary Tan, Alfonso R Herrera. St Luke's Hospital, Kilkenny, Ireland

\subsection{6/archdischild-2019-epa.566}

Introduction Human Bocavirus (HBoV) is a parvovirus first detected in 2005, being the most frequent pathogen responsible for respiratory tract infection in hospitalized children. The virus is detected most frequently in children less than 2 years old, but the $\mathrm{HBoV}$ infection is worldwide and that nearly all adults are seropositive. There is evidence of some antigenic cross reactivity among these viruses, and the biological significance of the different types is not clear.

We created this retrospective study due to increased days of hospitalization and need of oxygen therapy observed on the pediatric ward of our hospital during July 2017- July 2018 and we compared children that tested $\mathrm{HBoV}$ positive with those that tested $\mathrm{HBoV}$ negative on the extended viral swabs.

Objectives

1. To assess the length of hospitalization days.

2. To assess the need of $\mathrm{O}_{2}$ in our cohort patients.

3. To assess the prescription of chest $\mathrm{X}$-rays.

4. To assess the use of antibiotic-therapy.

5. To assess correlation with other viruses.

Population and method A total number of 60 extended viral swabs taken during July 2017- June 2018 were studied (13 $\mathrm{HBoV}$ positive and $47 \mathrm{HBoV}$ negative). Out of those 47 $\mathrm{HBoV}$ negative we selected 12 that correspond with the inclusion criteria. The method of testing used was PCR on extended nasal viral swabs. Inclusion criteria: respiratory infections and age similarity between the patients $\mathrm{HBoV}$ positive and $\mathrm{HBoV}$ negative in the set up period of time. Exclusion criteria: other diagnoses than the respiratory infections. 